\title{
Bioanalysis
}

\section{5th JBF Symposium: 'Bridging for a Global Harmonization'}

\section{Tower Hall Funabori, Tokyo, Japan, 6-7 March 2014}

At the 5th Japan Bioanalysis Forum symposium, distinguished bioanalytical scientists from government and industry commentated on the bioanalytical method validation guidelines/guidance issued by their own regions, including the draft bioanalytical method validation guideline for ligand binding assay by the Japanese Ministry of Health, Labour and Welfare, which was finalized in April 2014. Additionally, the Japan Bioanalysis Forum Discussion Group, in which daily bioanalytical issues/interests were scientifically discussed, picked up five topics, and more than 200 conference attendees openly exchanged their views on a wide range of bioanalytical issues with discussion group members. This manuscript provides an overview of the highlights out of the symposium.

Recently, bioanalytical method validation (BMV) guidelines were issued from various regions in a row, and every bioanalyst in the world was pressed with interpretations and differences of each BMV guideline. The Japan Bioanalysis Forum (JBF), established in 2011, exercised a vital role in the development of the BMV guidelines by the Japanese Ministry of Health, Labour and Welfare (MHLW). The BMV guideline on LC-MS/MS and HPLC (MHLW LC GL) was released by the MHLW in July 2013 [1], and subsequently the BMV guideline on Ligand Binding Assay (MHLW LBA GL) was issued in April 2014 [2]. Meanwhile, the US FDA issued draft BMV guidance in September 2013 [3], and the guidance had a big impact on regulated bioanalysis. Accordingly, the 5th JBF symposium is considered to be an opportune moment to cover the BMV guideline/guidance, including the the guideline issued by EMA (EMA GL) [4].

After a brief JBF activity report, with an understanding of the guidance/guideline, invited speakers gave a clear expositions of each BMV guidance/guideline. Additionally, we discussed perspectives on the harmonization of regulated bioanalysis, and LC-MS-based approaches for bioanalysis of biopharmaceuticals on panel discussion. As another topic, JBF discussion groups (DGs) officially initiated their activities and the outcomes were presented at the symposium. DGs were introduced at the 4th JBF symposium [5], and the main objective of the DGs is to discuss specific bioanalytical topics within small expertise groups.

This report provides an overview of the symposium's highlights and presentation materials are available on the JBF website [6].

\section{JBF activity report}

After opening remarks by Haruhiro Okuda (JBF; National Institute of Health Science [NIHS]; Tokyo, Japan), Masanari Mabuchi (JBF; Mitsubishi Tanabe Pharma, Osaka, Japan) presented updates on the JBF's activities after the 4th JBF symposium, held in August 2013.

First, Mabuchi showed the scope, mission, and organization of the JBF, andafter that, he looked back on previous JBF symposia [5,7]. In addition, Mabuchi mentioned JBF's future objectives. The JBF steering
Nobuhiro Kobayashi

DaiichiSankyo Co., Ltd 1-2-58, Hiromachi, Shinagawa-ku, Tokyo 140-8710, Japan Tel.: +81334923131 Fax: +8135740 3625 kobayashi.nobuhiro.ha@daiichisankyo. co.jp 
committee would commit to expanding DGs, releasing a handbook for the MHLW LC GL, follow-up MHLW LBA GL and upcoming conferences in which JBF would be engaged.

\section{Overview \& update of MHLW guidelines}

Noriko Katori (JBF; NIHS) presented activities for MHLW LC GL and MHLW LBA GL after a perspective of regulated bioanalysis and the BMV study group in Japan [8]. In addition, she introduced the large molecular LC-MS working group (leader: Nana Kawasaki; NIHS) consisting of 15 researchers from authorities and industry. Target analytes are therapeutic large molecules, such as protein, peptide, antibody-drug conjugate and nucleic acid, whereas biomarkers are out of scope. Their first goal is to provide any deliverables, which were not limited to guidelines.

They are going to try to clarify differences in guidance criteria of the LC-MS method between large and small molecules.

\section{MHLW LC GL}

Hiroko Miyai (Japan Pharmaceutical Manufacturers Association [JPMA]; Wakamoto Pharma, Kanagawa, Japan) presented survey results regarding regulatory compliance to MHLW LC GL in pharmaceutical companies.

MHLW LC GL became effective on 1 April 2014, while all pharmaceutical companies respond adequately to the prescriptions of the guideline without any major standard operating procedure revision. It would appear that most pharmaceutical companies have already consolidated their standard operating procedures based on the EMA GL and/or FDA guidance issued in 2001 [9], and MHLW LC GL did not make a significant change to EMA GL. Meanwhile, they recognized some differences among the EMA GL, the FDA guidance (2001) and the Japan guideline, and the differences are of increasing concern regarding global research and development. Therefore, they are keen to harmonize BMV internationally.

\section{Draft MHLW LBA GL}

Akiko Ishii (Chief of the LBA working group; NIHS) presented an overview of draft MHLW LBA GL. Seven members from JBF, two members from JPMA, five members from NIHS and one member from MHLW make up the working group for MHLW LBA GL.

Takahiro Nakamura (JBF; Shin Nippon Biomedical Laboratories, Kagoshima, Japan) then showed summary of draft MHLW LBA GL [10]. Several key principles of the guideline were as follows: the guideline also applies to small molecular drugs that are analyzed by LBAs; it is necessary to show well-established characteristics of reference standard; a full validation is also required for assays using a commercialized kit; minimum required dilution should be determined before a full validation; specificity should be evaluated; evaluation method for selectivity differs from that of MHLW LC GL; accuracy and precision should be evaluated also in ULOQ, and total error should be evaluated; concentration of high-level quality control (QC) is at least one-third of ULOQ; dilutional linearity should be evaluated; and, partial validation should be performed when changing a critical reagent lot or minimum required dilution.

As was mentioned at the beginning, MHLW LBA GL was finalized in April 2014. The team deserves great praise for finalizing the guideline in a short period.

\section{Perspectives of FDA draft guidance (2013) \& EMA GL}

Brian Booth (Office of Clinical Pharmacology, FDA, MD, USA) presented FDA draft guidance (2013) [3] focused on their philosophy in the revision and their feedback. As their philosophy, the FDA guidance should be neither prescriptive nor conceptual, and the guidance describes the issues that need to be satisfied for a validated method and the minimum standards. Additionally, the guidance allows for scientific judgment since prescriptive may be too restrictive, and they cannot conceive and capture all situations. Booth also emphasized some challenges in the guidance, namely, biomarkers and diagnostic kits; dried blood spot and Grafing other new technologies; and, cross/partial validations. Booth then answered some questions previously submitted by the JBF. Finally, he noted the FDA guidance revision. The docket consisted of 640 pages from around the world, and it was unclear when the FDA would complete the time-consuming process for the docket, and subsequently revise or finalize the FDA guidance.

Binodh DeSilva (American Association of Pharmaceutical scientists [AAPS]; Bristol-Myers Squibb, NJ, USA) presented summary and consensus from the AAPS workshop on Crystal City V Meeting [11] focused on LBA. She also noticed draft MHLW LBA GL and she complimented the guideline on the clarification of specificity and selectivity.

Michaela Golob (the European Bioanalysis Forum [EBF], Merck Serono, Grafing, Germany) presented EMA GL [4] focused on LBA, and she clarified not only the special requirement for LBA in the EMA GL [12], but also the differences from the draft FDA guidance (2013).

\section{Panel discussion (Theme: Harmony - 'All we have to do is dream')}

Prior to the panel discussion, Manish Yadav (APAIndia, ALKEM Labs, India) presented the current 
status of regulated bioanalysis in India. Bioanalytical activities in India are activated by participating in the Global Bioanalysis Consortium (GBC), and APA-India, consisting of more than 20 bioanalytical experts, was established.

Shinobu Kudoh (JBF; Shimadzu Techno Research, Kyoto, Japan) hosted a panel discussion, focusing on LC-MS of large molecules and harmonization of regulated bioanalysis. Panelists were Mark Arnold (AAPS; Bristol-Myers Squibb, NJ, USA), Binodh DeSilva, Michaela Golob, Daniel Tang (the China Bioanalysis Forum; Icon Development Solutions, China), Peter van Amsterdam (EBF; Abbott, Weesp, The Netherlands), Manish Yadav, Mami Imazato (Novartis Pharma, Tokyo, Japan), and Ryoya Goda (DaiichiSankyo, Tokyo, Japan).

\section{Perspectives on the harmonization of regulated bioanalysis in general}

Ideally, one global guideline or at least globally harmonized guidelines for BMV (large and small) should be agreed, but the reality is that a number of BMV guidelines have been published around the world in the past 2 years. The majority of the guidelines are similar, but there are still some discrepancies and different specific requirements. New areas, such as LBA, biomarkers and new technologies, are still to be defined. To build common understanding of regulated bioanalysis, one global guideline has been eagerly awaited. To fulfill it, a common platform it is important to continue discussion and agree on scientific basis for BMV globally within the bioanalysis (BA) community, but also with agencies. As there is more than one right answer, to strengthen scientific discussions and dialogue among bioanalytical and regulatory communities becomes more important to achieve harmonization and mindset change amongst stakeholders.

\section{LC-MS-based approaches for bioanalysis of biopharmaceuticals}

LC-MS-based approaches for bioanalysis of biopharmaceuticals have been a hot topic and there has been rapid growth with some successfully achieved cases in regulated bioanalysis in Europe and the USA with the expectation of a wide determination range, rapid outcomes and utility in answering questions that LBAs cannot. By contrast, there are still many technical hurdles (i.e., what type, a selected peptide or whole protein, as an internal standard should be prepared and how sensitivity and limited MW range over LBA are overcome). Many innovative approaches, such as acid separation, immunoextraction, selective interference removal technique, derivatization, 2D-chromatography, high-resolution MS and so on, have been introduced into its practice. In addition to their scientific expectations and considerations, the panelists also shared the general status of activities and initiatives with the approach in their own countries and communities, of which we understood there were big differences in companies as well as regions.

\section{Current \& future perspectives of the Global Bioanalysis Consortium on harmonization of bioanalytical guidance}

Peter van Amsterdam presented a status update on the Global Bioanalysis Consortium (GBC). After reviewing the timeline of activities, van Amsterdam showed the achievements of the GBC [13-16], namely bringing together a global community of bioanalytical scientists and stimulating regional discussions on bioanalytical guidance; visualizing the challenges of different global guidance; highlighting the need/desire for a harmonized (view on) bioanalytical regulations; stimulating best practices, considering science and regulations. Indeed, the GBC triggered a foundation of the JBF. In addition, many bioanalytical scientists joined the discussions in the GBC meeting, and obtained a lot of information. Meanwhile, he mentioned we have some challenges to accomplish global harmonization of bioanalysis, especially full consensus amongst regulators on a number of ambiguities. He also inquired whether GBC can grow to become the glue between regional bioanalysis organizations. It would perhaps be a good occasion that GBC activities are escalated to an international scientific bioanalytical community influencing cross-regional regulations, such as ICH, OECD or WHO.

\section{DG}

Yoshihisa Sano (JBF; Eisai, Tsukuba, Japan) presented an overview of the DG. The DG was founded under the JBF umbrella to provide opportunities for discussing issues raised from daily bioanalytical work among Japanese scientists, and topics are not limited to regulatory matters. After trials of which outcomes were presented at the previous JBF symposium, five DGs have officially initiated their activities and the outcomes were presented at the 5th JBF symposium.

Each leader of the five DGs presented an overview in the oral session, while all DG members showed their outcomes and held active discussion in the poster session. Since most DGs have plans to submit their deliverables, introduction of each DG was minimized.

DG1 dealt with the topic of 'preparation of calibration standard and QC samples'. The group consisted of 11 members (Leader: Harue Igarashi; JBF; GlaxoSmithKline, Toykyo, Japan) and investigated the practical procedures to prepare calibration standard and QC samples in each bioanalytical study in 
Japan in order to understand the distribution and diversity of the procedure. Besides, DG1 tried to recognize the features of the different procedures and think of the significance of the prepared samples, towards the proposal of recommendation.

DG2 took up the topic of 'Recommendation to prepare standard solutions', to propose a recommendation on preparation of standard solution as a Japanese standard based on scientific discussion. The group consisted of nine members (Leader: Takahiko Osumi; JBF; Otsuka,Tokushima, Japan). The topic has been taken up at the trial DG, and DG2 tried to explore the best way to prepare standard solutions in reference to the questionnaire at the 4th JBF symposium.

DG3 focused on the topic of 'Tiered approach for bioanalytical method of metabolites', so that we can understand the tiered approach from a practical point of view. The group consisted of eight members (Leader: Makoto Niwa; Nippon Kayaku, Tokyo, Japan). First, DG3 members ensured shared understanding of the 'tiers', and subsequently discussed how to use the given tier and reference standards in metabolite analysis. DG3 defined 'validated method', 'qualified method', 'research method' and 'screening method' as typical tiered approach. After that, DG3 showed recommended methods for analysis of metabolite both in preclinical and clinical phases. Additionally, DG3 members realized differences in perspective about quality of reference standards for metabolites.

DG4 covered the topic of 'Partial validation', focusing on change in matrix. The group consisted of $13 \mathrm{mem}-$ bers (Leader: Akemi Nagao; Japan Tobacco, Osaka, Japan). We cannot find any clear description of how partial validation should be done in BMV guidelines. Therefore, DG4 was initiated to clarify study design of partial validation, concentrating on 'change in plasma', since the topic was the most interesting modification in the validated bioanalytical method among DG4 members. They discussed validation items to be evaluated on change based on the survey of JBF members. They presented typical methodologies for some specific cases.

DG5 devoted sufficient effort to a versatile topic on LBA including PK, anti-drug antibody and biomarker assays. The group consisted of 11 members (Leader: Kazuhiro Miya: JBF; Chugai, Toyko, Japan). DG5 agreed the batch of reference standard for QC

\section{References}

1 Japanese Ministry of Health, Labour and Welfare. Guideline on Bioanalytical Method Validation in Pharmaceutical Development. (11 July 2013, MHLW, Japan).

www.nihs.go.jp/drug/BMV/250913_BMV-GL_E.pdf and calibrator is not necessarily required to be identical to that for dosing formulation, while EMA GL strongly recommends they are identical [4]. As for anti-drug antibody assays in Japan, there are not any regulatory guidelines available at this moment in time; DG5 intensively discussed these assays. For example, DG5 favored the idea that binding assays can usually be used in neutralizing antibody assay instead of cellbased assays. DG5 had many more arguments, some of them formulated members' agreement and the others remained as future subjects. For instance, a very small cut-point due to small variance of individual samples is one of the practical problems. Finally, DG5 formed and moved into successive groups for the outstanding issues without any interruption and also for newly raised questions.

JBF decided to expand the DG activity and began to set up seven DGs, including new topics and members.

\section{Conclusion}

The BMV study group sponsored by MHLW, consisted of authorities and scientists from industry, has been working together toward multiple regulated bioanalytical activities, whereas we recognized that we need to overcome some obstacles to accomplish global harmonization of regulated bioanalysis.

The JBF has become activated as a sole forum on regulated bioanalysis in Japan, and the JBF plans to hold the 6th JBF symposium in Tokyo in February 2015.

\section{Acknowledgements}

As the symposium organizer, the author would like to thank the: JPMA Pharmacokinetics team for their informative presentations, symposium attendees and JBF DG members for their attentive discussion and the JBF steering committees for their dedication to the forum.

Financial \& competing interests disclosure

The author has no relevant affiliations or financial involvement with any organization or entity with a financial interest in or financial conflict with the subject matter or materials discussed in the manuscript. This includes employment, consultancies, honoraria, stock ownership or options, expert testimony, grants or patents received or pending, or royalties.

No writing assistance was utilized in the production of this manuscript.

2 Japanese Ministry of Health, Labour and Welfare. Guideline on Bioanalytical Method (Ligand Binding Assay) Validation in Pharmaceutical Development. (1 April 2014, MHLW, Japan). www.nihs.go.jp/drug/BMV/260530_LBA-GL_E.pdf

3 US Department of Health and Human Services, Food and Drug Administration. Draft Guidance for Industry: Bioanalytical Method Validation (September 2013). 
www.fda.gov/downloads/Drugs/

GuidanceComplianceRegulatoryInformation/Guidances/

UCM368107.pdf

4 European Medicine Agency. Guideline on bioanalytical method validation. EMEA/CHMP/EWP/192217/2009 (2011).

www.ema.europa.eu/docs/en_GB/document_library/ Scientific_guideline/2011/08/WC500109686.pdf

5 Nakayama A, Nakamura T. Conference Report: 4th Japan Bioanalysis Forum Symposium: Japanese guidelines on bioanalytical method validation and latest discussion within the Japanese bioanalyst community. Bioanalysis 6(7), 915-917 (2014).

6 Japan Bioanalysis Forum. http://bioanalysisforum.jp/en

7 Imazato M. Conference Report: International harmonization of bioanalysis regulation: discussion in Global Bioanalysis Consortium harmonization teams. Bioanalysis 5(3), 281-283 (2013).

8 Katori N. Regulated bioanalysis in Japan: where do we come from and where are we going? Bioanalysis 5(11), 1321-1323 (2013).

9 US Department of Health and Human Services, Food and Drug Administration. Guidance for Industry: Bioanalytical Method Validation (May, 2001). www.fda.gov/downloads/Drugs/Guidances/ucm070107.pdf

10 Japanese Ministry of Health, Labour and Welfare. Guideline on Bioanalytical Method (Ligand Binding Assay) Validation in Pharmaceutical Development (Draft) (10 January 2014, MHLW, Japan).
11 Smeraglia J, McDougall, Timmerman P et al. Conference Report: AAPS and US FDA Crystal City V meeting on Quantitative Bioanalytical Method Validation and Implementation: feedback from the EBF. Bioanalysis 6(6), 729-732 (2014).

12 van Amsterdam P, Companjen A, Brudny-Kloeppel M, Golob M, Luedtke S, Timmerman P. The EBF community's evaluation and interpretation of the EMA guideline on bioanalytical method validation towards its implementation Bioanalysis 5(6), 645-659 (2013).

13 Sailstad JM, Amaravadi L, Wustner JT et al. A white paper - consensus and recommendations of a global harmonization team on assessing the impact of immunogenicity on pharmacokinetic measurements. AAPS J. 16(3), 488-498 (2014).

14 Young GC, Seymour M, Dueker SR, Timmerman P, Arjomand A, Nozaka K. New frontiers-accelerator mass spectrometry (AMS): recommendation for best practices and harmonization from Global Bioanalysis Consortium Harmonization Team. AAPS J. 16(2), 357-359 (2014).

15 Dudal S, Baltrukonis D, Yang J et al. Assay formats: recommendation for best practices and harmonization from the global bioanalysis consortium harmonization team. AAPS J. 16(2), 194-205 (2014).

16 Ahene A, Calonder C, Wang J et al. Automation practices in large molecule bioanalysis: recommendations from group L5 of the global bioanalytical consortium. AAPS J. 16(1), 164-171 (2014). 